OPEN ACCESS

Edited by:

Quan Zou,

University of Electronic Science and Technology of China, China

Reviewed by: Heng Pan,

Cornell University, United States

Hao Lin,

University of Electronic Science and Technology of China, China

*Correspondence:

Hao Zhang

zhangh@jlu.edu.cn

Specialty section:

This article was submitted to Bioinformatics and Computational

Biology,

a section of the journal

Frontiers in Genetics

Received: 07 October 2018 Accepted: 15 November 2018 Published: 29 November 2018

Citation:

Zhong X, Liu Y, Liu H, Zhang Y, Wang $L$ and Zhang $H$ (2018) Identification of Potential Prognostic Genes for Neuroblastoma.

Front. Genet. 9:589.

doi: 10.3389/fgene.2018.00589

\section{Identification of Potential Prognostic Genes for Neuroblastoma}

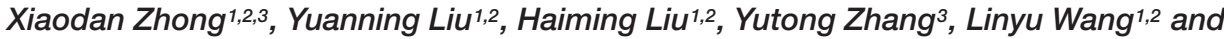 \\ Hao Zhang ${ }^{1,2 *}$ \\ ${ }^{1}$ College of Computer Science and Technology, Jilin University, Changchun, China, ${ }^{2}$ Key Laboratory of Symbolic \\ Computation and Knowledge Engineering, Ministry of Education, Jilin University, Changchun, China, ${ }^{3}$ Department \\ of Pediatric Oncology, The First Hospital of Jilin University, Changchun, China
}

Background and Objective: Neuroblastoma (NB), the most common pediatric solid tumor apart from brain tumor, is associated with dismal long-term survival. The aim of this study was to identify a gene signature to predict the prognosis of NB patients.

Materials and Methods: GSE49710 dataset from the Gene Expression Omnibus (GEO) database was downloaded and differentially expressed genes (DEGs) were analyzed using R package "limma" and SPSS software. The gene ontology (GO) and pathway enrichment analysis were established via DAVID database. Random forest (RF) and risk score model were used to pick out the gene signature in predicting the prognosis of NB patients. Simultaneously, the receiving operating characteristic (ROC) and Kaplan-Meier curve were plotted. GSE45480 and GSE16476 datasets were employed to validate the robustness of the gene signature.

Results: A total of 131 DEGs were identified, which were mainly enriched in cancer-related pathways. Four genes (ERCC6L, AHCY, STK33, and NCAN) were selected as a gene signature, which was included in the top six important features in RF model, to predict the prognosis in NB patients, its area under the curve (AUC) could reach 0.86 , and Cox regression analysis revealed that the 4-gene signature was an independent prognostic factor of overall survival and event-free survival. As well as in GSE16476. Additionally, the robustness of discriminating different groups of the 4-gene signature was verified to have a commendable performance in GSE45480 and GSE49710.

Conclusion: The present study identified a gene-signature in predicting the prognosis in NB, which may provide novel prognostic markers, and some of the genes may be as treatment targets according to biological experiments in the future.

Keywords: neuroblastoma, differentially expressed genes, gene signatures, prognosis, GEO, ERCC6L

\section{INTRODUCTION}

Neuroblastoma (NB) is a highly heterogeneous pediatric solid tumor both in clinical and biological characteristics. It is the third most common malignant disease, which takes about $7 \%$ of malignant tumors in children less than 14 years, with the incidence of close to 60/100,000 during the first year of life (Maris et al., 2007; Sausen et al., 2013; Ward et al., 2014). However, NB has occupied up to $15 \%$ of cancer-related death in childhood (Maris et al., 2007; Domingo-Fernandez et al., 2013; 
Sausen et al., 2013; Salazar et al., 2016; Stafman and Beierle, 2016). Different from adult cancers, NB presents a low frequency of mutation and rearrangement, which only takes up less than 40\% (Pugh et al., 2013). Multiple "omics" studies have found that some molecules are involved in NB development, including MYCN, ALK, LMO1, PHOX2B, ARID1A, and ARID1B (Huang and Weiss, 2013; Pugh et al., 2013; Sausen et al., 2013; Beckers et al., 2015; Bosse and Maris, 2016; Liu and Thiele, 2017). Nonetheless, the survival time of the high-risk group has not been markedly prolonged, with the long-term survival of less than 40 50\% (Maris et al., 2007; Pinto et al., 2015; Salazar et al., 2016).

Typically, patients at stage 4 and stage $4 \mathrm{~s}$ all have metastatic tumors, but they have remarkably different outcomes. Notably, most patients with stage 4 diseases are associated with high risks and inferior outcomes regardless of multi-modal therapy (Maris et al., 2007; Whittle et al., 2017). In contrast, stage $4 \mathrm{~s}$ diseases frequently occur in infants, and they generally have beneficial outcomes even though without any treatment or moderate chemotherapy (Rubie et al., 2011; Brodeur and Bagatell, 2014). Studies indicate that more than 20\% NB cases have experienced spontaneous remission or regression, especially for stage 4s cases (Diede, 2014; Attiyeh and Maris, 2015). In addition, some studies have found the differences between stage 4 and stage $4 \mathrm{~s}$ patients. For instance, Taggart et al. (2011) had accessed 0-18 months old patients with stage 4 and stage $4 \mathrm{~s}$ diseases, and found that the tumor biological features [such as MYCN, 11q, mitosis-karyorrhexis index (MKI), histology, and 1p] were more important than age and metastatic pattern for predicting the clinical outcomes, which should be considered for risk stratification in patients aged less than 18 months. Moreover, Fischer et al. (2006) had identified a special gene expression pattern between stage 4 and stage $4 \mathrm{~s}$ patients. Bénard et al. (2008) had identified a stage 4 s gene signature by comparing the gene expression profiles between stage 4 and stage $4 \mathrm{~s}$ patients. However, this is far from enough, and further studies are needed, which may provide some inspiration for understanding and exploiting the therapeutic strategies of NB.

Random forest (RF) is among the most important machine learning methods thanks to their relatively good accuracy, robustness, and ease of use. They can be used to rank the importance of features in a regression or classification problem in a natural way. Mean decrease impurity and mean decrease accuracy are employed as criteria for feature selection (Strobl et al., 2007).

In this paper, we have identified a four-gene signature via RF and risk score model in predicting the prognosis of $\mathrm{NB}$ patients via the GEO dataset (GSE49710), the gene signature also had good performance in discriminating other groups of GSE49710, as well as in other two independent datasets. Importantly, the obtained gene signature we picked out could separate NB patients with different outcomes in stage 4 or age less than 18 months, or MYCN not amplified. Thus, the gene signature was considered as a prognostic marker of $\mathrm{NB}$, and some genes (like ERCC6L) in the signature might serve as the therapeutic targets based on biological experiments in the future.

\section{MATERIALS AND METHODS}

\section{Data Collection and Processing}

Data were downloaded from GEO datasets GSE49710 (Supplementary Data Sheet S1), GSE45480, and GSE16476. Samples with the survival time of less than 30 days and more than 10 years were excluded. Finally, 419 samples were obtained, and 45 stage 4 patients with the survival time of less than 18 months as well as 50 stage $4 \mathrm{~s}$ patients were also employed in this study. R package "limma" (Ritchie et al., 2015) was used for data processing, and expression data were transformed by $\log 2$ calculation. Meanwhile, the prediction ability was validated using the GSE45480 (GPL16876) and GSE16476 datasets. The highest expression value was employed to represent the gene expression level when a single gene matched multiple probes. Eventually, the top $1 \%$ mRNAs with high expression were selected in stage 4 patients and the adjusted $p$-value of $<0.05$ was considered as differentially expressed genes (DEGs). Moreover, log-rank test and Cox proportional hazard regression model (Prentice, 1992) were utilized to identify the risk factors related to clinical prognosis. Genes with a hazard ratio of $>1$ and a $p$-value of less than 0.001 upon univariate Cox regression were picked out.

\section{Functional Enrichment Analysis of DEGs}

Functional enrichment analysis of the candidate DEGs was carried out using the online database DAVID 6.8 ${ }^{1}$. In the meantime, gene ontology (GO) (The Gene Ontology Consortium, 2017) term BP (Biological Process) and Kyoto Encyclopedia of Genes and Genomes (KEGG) (Kanehisa et al., 2017) analysis were performed to provide gene biological function, with $p<0.05$ as the cut-off criterion.

\section{Selection of Gene Signature and Confirmation of Performance}

Firstly, we put the DEGs into the RF model as features, then built the RF model using Scikit-Learn tools. We defined a grid of hyperparameter, and sampled from the grid, performing 10-fold cross-validation with each combination of values. Subsequently, the grid search algorithm outputs the settings that achieved the highest AUC in the validation procedure and the feature importance based on mean decrease impurity (Strobl et al., 2007). Secondly, based on the above ranking results, we combined top 10 important genes to predict the prognosis of patients, then selected one of the optimal combinations, and the coefficient of candidate genes was also calculated with multivariate Cox regression analysis. Thirdly, the risk score model (Zhou et al., 2015; Liao et al., 2018) was adopted to evaluate the performance in predicting the vital status and assess its discrimination ability in other different groups. In addition, the receiving operating characteristic (ROC) curve was plotted.

$$
\text { Risk Score }=\sum_{i=1}^{n} \beta_{i}^{*} \chi_{i}
$$

\footnotetext{
${ }^{1}$ https://david.ncifcrf.gov/
} 
where $\beta_{\mathrm{i}}$ indicated the coefficient of each gene in multivariate Cox regression analysis with overall survival as the dependent parameter and $\chi_{i}$ represented the expression value by $\log 2$ transformation of each gene.

Simultaneously, all samples were divided into the high or low-risk group according to the median risk score, and the Kaplan-Meier curves of overall survival and event-free survival were plotted.

\section{Statistical Analysis}

All statistical analyzes were carried out using the IBM SPSS version 23 software and R. Bivariate coefficient was calculated through Spearman's rank correlation. Additionally, Kaplan-Meier method and log-rank test were employed to plot and compare the survival curves. Survival data were assessed through univariate and multivariate Cox regression analyses. A two-tailed $p$-value of $<0.05$ was considered statistically significant.

\section{RESULTS}

\section{Clinical Characteristics Analysis of GEO Data}

A total of 498 NB samples with complete clinical data were downloaded from the GSE49710 dataset, and 419 of them were finally included in our study. The details of clinical/pathological features were listed in Table 1. Survival analysis showed that INSS stage 4 patients had inferior outcomes to those of stage 4 s patients (Supplementary Figure S1). Stage 4 patients had taken up 63.6, 70.0, 77.7, and 83.9\% among all the 419 patients with progression, MYCN amplification, death from disease and high risk, respectively. In contrast, stage 4s patients with MYCN amplification, high risk and death from disease had occupied less than $4.5 \%$, and those with progression had accounted for $7.5 \%$. In comparison, in the TARGET-NBL dataset, stage 4 patients had taken up more than $80 \%$ in death, event, progression and relapse groups, and the proportions were especially high in death and relapse groups (96.9, and 91.3\%, respectively). However, stage $4 \mathrm{~s}$ patients had only occupied no more than 2\% (Supplementary Table S1). These results demonstrated huge differences between stage 4 and stage $4 \mathrm{~s}$ patients. Cox regression analysis of stage 4 patients (GSE49710, $n=168$ ) revealed that the age of $\geq 18$ months, MYCN amplification, and high risk were associated with inferior outcomes [hazard ratio: 2.237 (95\% CI: 1.209-4.141), 2.437 (95\% CI: 1.557-3.814), 11.208 (95\% CI: 2.746-45.743) and p-value: 0.01, < 0.001, 0.001, respectively]. Results of risk factor analysis in this dataset were consistent with those from related articles (Pinto et al., 2015).

\section{Identification of DEGs and Functional Enrichment Analysis in NB}

In this study, 45 INSS stage 4 patients aged less than 18 months and 50 stage $4 \mathrm{~s}$ patients were enrolled from the GSE49710
TABLE 1 | Clinical characteristics of NB patients in GSE49710 (samples with survival time $\leq 30$ days and $\geq 10$ years have been removed).

\begin{tabular}{|c|c|}
\hline Characteristics & Number of cases (\%) \\
\hline \multicolumn{2}{|l|}{ Gender } \\
\hline Male & 247 (58.9) \\
\hline Female & 172 (41.1) \\
\hline \multicolumn{2}{|l|}{ Age at Diagnosis } \\
\hline$<18$ months & 246 (58.7) \\
\hline$\geq 18$ months & 173 (41.3) \\
\hline \multicolumn{2}{|l|}{ MYCN Status } \\
\hline Amplified & $90(21.5)$ \\
\hline Not Amplified & 324 (77.3) \\
\hline $\mathrm{N} / \mathrm{A}$ & $5(1.2)$ \\
\hline \multicolumn{2}{|l|}{ Risk Group } \\
\hline High risk & $168(40.1)$ \\
\hline Intermediate or low risk & 251 (59.9) \\
\hline \multicolumn{2}{|l|}{ INSS Stage } \\
\hline 1 & $90(21.5)$ \\
\hline 2 & $65(15.5)$ \\
\hline 3 & $46(11.0)$ \\
\hline 4 & $168(40.1)$ \\
\hline $4 s$ & $50(11.9)$ \\
\hline \multicolumn{2}{|l|}{ Class Label } \\
\hline Favorable & $141(33.7)$ \\
\hline Unfavorable & $90(21.5)$ \\
\hline $\mathrm{N} / \mathrm{A}$ & 188 (44.9) \\
\hline \multicolumn{2}{|l|}{ Progression } \\
\hline Yes & 173 (41.3) \\
\hline No & $246(58.7)$ \\
\hline \multicolumn{2}{|l|}{ Death from Disease } \\
\hline Yes & 103 (24.6) \\
\hline No & 316 (75.4) \\
\hline
\end{tabular}

N/A, not applicable; Class label, Maximally divergent disease courses; unfavorable, patients died despite intensive chemotherapy; favorable, patients survived without chemotherapy for at least 1000 days post-diagnosis.

dataset, so as to exclude the influence of age. According to the cut-off criterion of adjusted $p$-value of $<0.05$, the top $1 \%$ high expression genes of 26082 mRNAs in stage 4 patients were selected, and a total of 215 differentially expressed mRNAs were identified after excluding the repetitive genes. Afterward, genes with the hazard ratio of $>1$ and $p$-value of $<0.001$ upon univariate Cox regression were chosen, and 131 DEGs were selected at last (Supplementary Table S2).

The functions and pathway enrichment of the candidate DEGs were analyzed using online database DAVID, and 58 GO terms in BP (Biological Process) and 5 KEGG pathways were revealed finally (Figures $\mathbf{1 A}, \mathbf{B}$ and Supplementary Tables S3, S4). As shown in Figure 1A, the DEGs were mainly enriched in cell division, DNA replication, mitotic nuclear division, DNA repair and cell proliferation (Stafman and Beierle, 2016). In terms of KEGG pathways, the DEGs were mainly enriched in cell cycle (Williams and Stoeber, 2012; Otto and Sicinski, 2017), Fanconi anemia pathway (Ceccaldi et al., 2016), pyrimidine metabolism (Kelemen et al., 2014), 
A

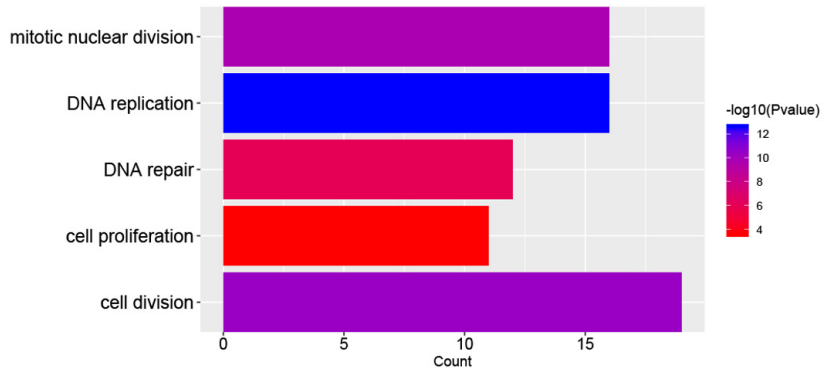

B

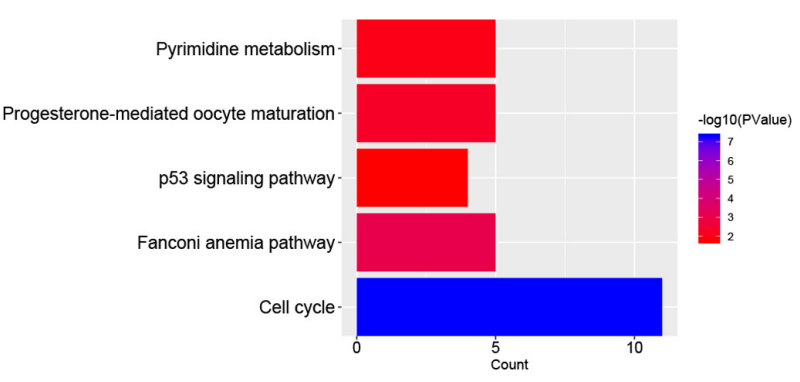

FIGURE 1 | Functional enrichment analysis of DEGs in NB. (A) The top 5 gene count of gene ontology (GO) terms in NB-related genes; (B) The top 5 significant KEGG pathways in NB-related genes.

and p53 signaling pathways, which were the cancer-related pathways.

\section{Performance of Gene Signature in Predicting Prognosis}

In order to select the genes with good performance in predicting prognosis of NB, RF was adopted to rank the 131 DEGs in predicting the prognosis in NB patients (Supplementary Table S5). Based on the ranking results, four genes (ERCC6L, $A H C Y, S T K 33$, and NCAN) were selected as a gene signature in predicting the vital status of NB patients (Table 2). To integrate four genes, multivariate Cox regression analysis was employed to obtain the coefficient. The risk score was calculated as follows, risk score $=$ expression of ERCC6L $L^{*} 0.408$ + expression of $A H C Y^{*} 0.478+$ expression of $S T K 33^{*} 0.345$ + expression of $N C A N^{*} 0.136$. Notably, the AUC of the 4-gene signature in predicting vital status could reach 0.86. Simultaneously, the 4-gene signature was employed to distinguish different groups, including $M Y C N$ amplification vs. Non-amplification, high risk vs. non-high risk, and progression vs. Non-progression. According to the ROC curve, the AUC of MYCN amplification, high risk, and progression reached 0.965, 0.928, and 0.78, respectively, as shown in Figure 2. Surprisingly, AHCY performed excellently in predicting MYCN amplification, with an AUC of 0.946 (Supplementary Table S6).

\section{Association of 4-gene Signature and Clinical Characteristics}

Four genes (ERCC6L, AHCY, STK33, and NCAN) were closely correlated with overall survival and event-free survival. As shown in Supplementary Figures $\mathbf{S 2 A - H}$, the increased expression of the four genes was linked with markedly shorter survival time. In risk score model, the group with high risk score of four gene-signature had significant inferior outcomes in NB patients (Figures 3A,B). Meanwhile, the risk score in different groups was calculated, and the results revealed that a high age, MYCN amplification, advanced stage, high risk, disease progression, and unfavorable class label groups had high risk scores $(p<0.0001)$ (Figure 3C). Moreover, the overall survival and event-free survival were further assessed in stage 4 patients, as well as those with $M Y C N$ non-amplification, and age of less than 18 months with high/low risk score. The results showed that the high-risk group had significant short overall and event-free survival time (Figure 4).

Spearman analysis of the correlation between 4-gene signature and clinicopathological features revealed that the high risk score group showed markedly positive correlation with age, risk, and INSS stage, while negative correlation with overall survival and event-free survival time. In addition, the high risk score group was also associated with unfavorable class label and MYCN amplification, as summarized in Table 3. Taken together, these known risk factors of the prognosis of NB (Pinto et al., 2015) were

TABLE 2 | Top 10 genes with high Gini importance by the random forest algorithm.

\begin{tabular}{|c|c|c|c|c|c|c|c|c|}
\hline Accession no. & Gene symbol & Gini importance & $\log F C$ & adj.P.Value & Chr & HR (OS) & $95 \% \mathrm{Cl}$ & $P$-value \\
\hline NM_004386 & NCAN & 0.061850062 & 1.697 & 0.002175968 & chr19 & 1.475 & $1.345-1.617$ & $<0.0001$ \\
\hline NM_000687 & AHCY & 0.038477178 & 0.686 & 0.000319358 & chr20 & 3.131 & 2.494-3.932 & $<0.0001$ \\
\hline NM_030906 & STK33 & 0.034351926 & 1.202 & 0.001117677 & chr11 & 1.798 & $1.569-2.061$ & $<0.0001$ \\
\hline NM_014501 & UBE2S & 0.028596074 & 0.84 & 3.01E-05 & chr19 & 2.943 & 2.355-3.679 & $<0.0001$ \\
\hline NM_003686 & EXO1 & 0.021770529 & 0.953 & 0.001977921 & chr1 & 2.148 & $1.811-2.548$ & $<0.0001$ \\
\hline NM_017669 & ERCC6L & 0.020307985 & 0.952 & 0.000570824 & $\operatorname{chrX}$ & 2.294 & $1.920-2.741$ & $<0.0001$ \\
\hline NM_016354 & SLCO4A1 & 0.018810664 & 1.747 & 0.001106007 & chr20 & 1.463 & $1.341-1.597$ & $<0.0001$ \\
\hline NM_001827 & CKS2 & 0.018428233 & 0.678 & 0.004725484 & chr9 & 2.679 & $2.179-3.294$ & $<0.0001$ \\
\hline NM_002358 & MAD2L1 & 0.018307845 & 0.777 & 0.007867488 & chr4 & 2.082 & $1.744-2.486$ & $<0.0001$ \\
\hline NM_032117 & MND1 & 0.018163659 & 0.849 & 0.002682907 & chr4 & 2.381 & $1.985-2.885$ & $<0.0001$ \\
\hline
\end{tabular}

Bold values indicate the genes we selected as the signature. 

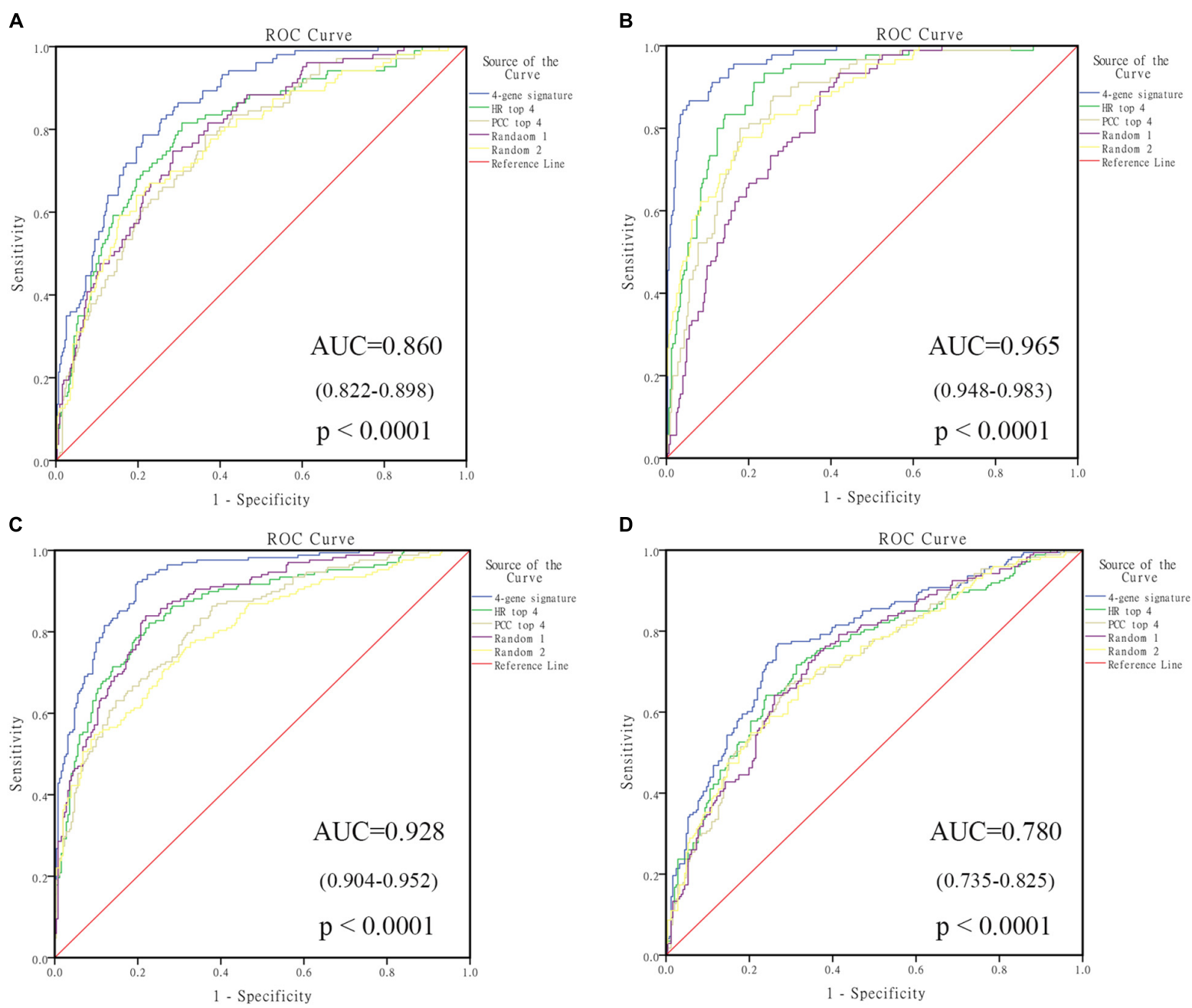

FIGURE 2 | The receiving operating characteristic curve (ROC) of GSE49710. (A) ROC of vital status; (B) ROC of MYCN amplified; (C) ROC of high risk; (D) ROC of progression. HR, hazard ratio; PCC, Pearson correlation coefficient.

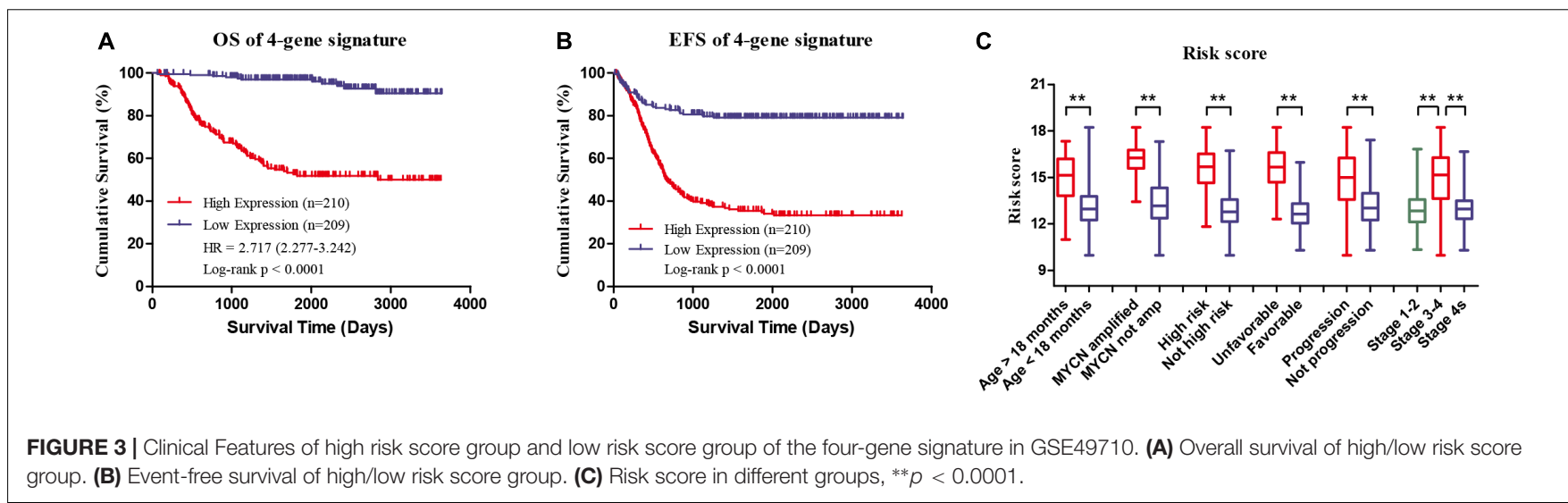

consistent with the 4-gene signature-based risk score selected in this study.

Univariate analysis suggested that the 4-gene signature, age group (age of $\geq 18$ months and age of $<18$ months), MYCN amplification, high risk, and INSS stage were the markedly prognostic factors of the overall survival and event-free survival in NB (all ps < 0.0001) (Table 4). Besides, multivariate survival analysis was also performed using the variables with significance 

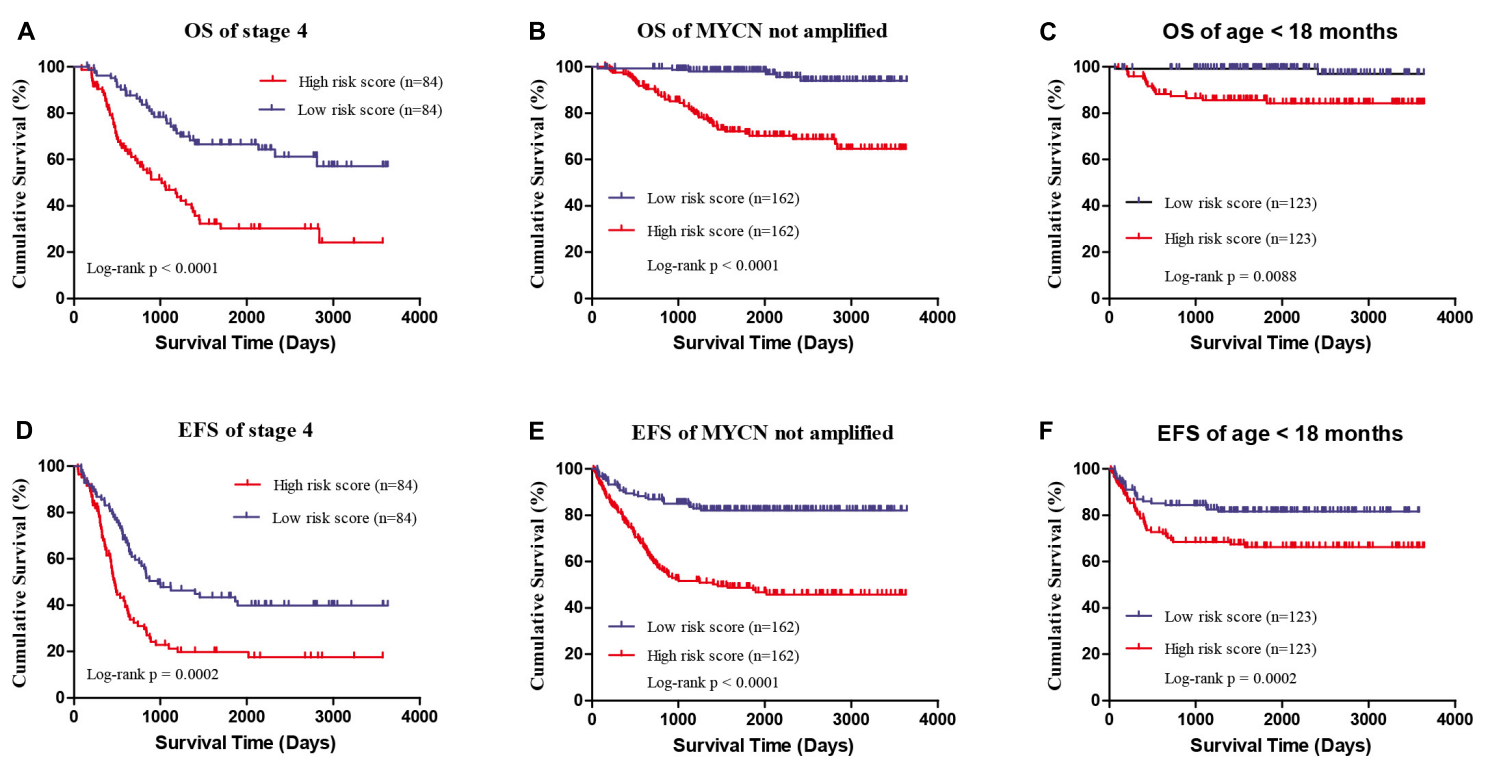

FIGURE 4 | The survival analysis of high/low risk score group of the four gene signature with exclusion of known risk factors. (A,D) OS and EFS in stage 4 patients. (B,E) OS and EFS of MYCN not amplified patients. (C,F) OS and EFS of age less than 18 months patients.

TABLE 3 | Spearman analysis of correlation between four-gene signature and clinicopathological features in GSE49710.

\begin{tabular}{lcc}
\hline Variables & \multicolumn{2}{c}{ Risk score group } \\
\cline { 2 - 3 } & spearman correlation & $P$-value \\
\hline Age at diagnosis & 0.530 & $<0.0001$ \\
MYCN amplified & 0.577 & $<0.0001$ \\
High Risk & 0.714 & $<0.0001$ \\
INSS stage & 0.588 & $<0.0001$ \\
Class label & 0.767 & $<0.0001$ \\
Progression & 0.473 & $<0.0001$ \\
Death from disease & 0.533 & $<0.0001$ \\
EFS days & -0.367 & $<0.0001$ \\
OS days & -0.362 & $<0.0001$ \\
\hline
\end{tabular}

in univariate analysis. The results confirmed that the 4-gene signature and INSS stage remained independent prognostic indicators for unfavorable overall survival and event-free survival (Table 4).

\section{Confirmation of Potential Function of ERCC6L in NB}

In the four-gene signature, the AUC of ERCC6L reached 0.799 in predicting the survival status of NB patients, which was the highest. In order to obtain the potential function of $E R C C 6 L$ in NB development, the gene-encoded protein-protein interaction (PPI) network was constructed by employing the STRING database ${ }^{2}$, so as to find the co-expression relationship of genes. CytoHubba (Chin et al., 2014) and MCODE (Bader and Hogue, 2003) of Cytoscape (Shannon et al., 2003) software were employed to screen the hub genes that interacted with ERCC6L, and 8 genes (including MAD2L1, NDC80, CCNB1, KIF18A, BIRC5, CENPM, CDCA5, and CCNB2) with the Clustering Coefficient of $>0.9$ were selected, as shown in Figure 5A. And the Pearson correlation coefficient (PCC) of the nine genes was also calculated. As shown in Figure 5B, the PCC of every two genes was higher than 0.8 ( $p$-value $<2.2 \mathrm{E}-16)$ (PCC of all DEGs in Supplementary Table S8). The above results suggested

${ }^{2}$ https://string-db.org/

TABLE 4 | Univariate and multivariate Cox regression analysis of the correlation between the 4-gene signature and clinical features in GSE49710.

\begin{tabular}{|c|c|c|c|c|c|c|}
\hline \multirow[b]{2}{*}{ Variables } & \multicolumn{3}{|c|}{ Univariate analysis } & \multicolumn{3}{|c|}{ Multivariate analysis } \\
\hline & $P$-value & Hazard ratio & $95 \% \mathrm{Cl}$ & $P$-value & Hazard ratio & $95 \% \mathrm{Cl}$ \\
\hline Age group & $<0.0001$ & 7.634 & $4.678-12.458$ & 0.098 & 1.716 & $0.904-3.256$ \\
\hline MYCN amplified & $<0.0001$ & 6.001 & $4.048-8.896$ & 0.166 & 1.386 & $0.874-2.198$ \\
\hline High risk & $<0.0001$ & 16.643 & $9.274-29.868$ & 0.323 & 1.666 & $0.606-4.583$ \\
\hline
\end{tabular}

Overall survival. Bold values emphasize $p<0.05$. 
that ERCC6L might interact with one or more genes in the development of NB. Nevertheless, the mechanism of ERCC6L in NB should be further confirmed from biological experiments.

\section{Validation of Performance in Other NB Datasets}

To confirm the robustness of the 4-gene signature in predicting different groups, two independent datasets GSE16476 and GSE45480 (GPL16876) were used for evaluation. As shown in Figure 6, the 4-gene signature had a commendable performance in dividing MYCN amplification and non-amplification groups, with the AUC of 0.964 and 0.975 , respectively. Meanwhile, the AUC of the 4-gene signature was 0.896 and 0.888 , respectively, in the vital status prediction of GSE16476 and discrimination of stage 4 and stage $4 \mathrm{~s}$ in GSE45480. Through analyzing the association of 4-gene signature with clinical features in GSE16476, we obtained similar results as GSE49710 (Supplementary Figures S3, S4 and Supplementary Table S7). We divided patients into high/low risk score group based on the median of 4-gene signature, the results showed that high risk score group correlated with age more than 18 months, advanced stage, MYCN amplified, and disease recurrence or progression, and had negative correlation with follow-up time.

\section{DISCUSSION}

$\mathrm{NB}$, an embryonal malignant tumor during early childhood, will lead to poor long-term survival, especially for stage 4 patients. Stratified treatment has achieved great progress in the past two decades (Pinto et al., 2015; Whittle et al., 2017); in the meanwhile, MYCN amplification and $A L K$ mutation have been comprehensively studied as the prognostic factors and important treatment targets (Barone et al., 2013; Huang and Weiss, 2013; Beckers et al., 2015; Liu and Thiele, 2017). However, MYCN amplification accounts for only about $25 \%$ patients (Huang and Weiss, 2013). Consequently, identifying key genes, exploring new targets for therapy, and ultimately improving survival time of patients with inferior outcomes remain challenging.

This study had focused on discriminating NB with tremendously different clinicopathological features, identifying the differentially expressed genes (DEGs) in the untreated stage 4 and stage $4 \mathrm{~s}$ patients, and utilizing bioinformatic means to intensively analyze the data. We had identified 131 DEGs, and BP in GO term and KEGG functional enrichment analysis showed that the DEGs were mainly enriched in the cancer-related pathways, such as DNA replication, cell division and cell cycle (Williams and Stoeber, 2012; Otto and Sicinski, 2017). We picked out a four-gene (ERCC6L, AHCY, STK33, and NCAN) signature in predicting the prognosis of NB, with the AUC of as high as 0.86 . Simultaneously, the grouping ability of the gene signature was assessed, the results of which revealed that the four-gene signature had good performance in predicting other groups. When predicting the MYCN amplification group, the AUC of the four-gene signature was 0.965 , and in another two independent datasets, the AUC in predicting MYCN amplification was higher than 0.95. We guessed there are two reasons: (1) the AUC in predicting $M Y C N$ amplification of $A H C Y$ alone was 0.946 , which could be attributed to the fact that $A H C Y$ was directly regulated by MYC proteins (Chayka et al., 2015); (2) might be due to the insufficient sample size and imbalanced distribution of positive and negative samples.

An ideal prediction model should help clinicians to determine optimal treatment strategies or aid them to predict patient
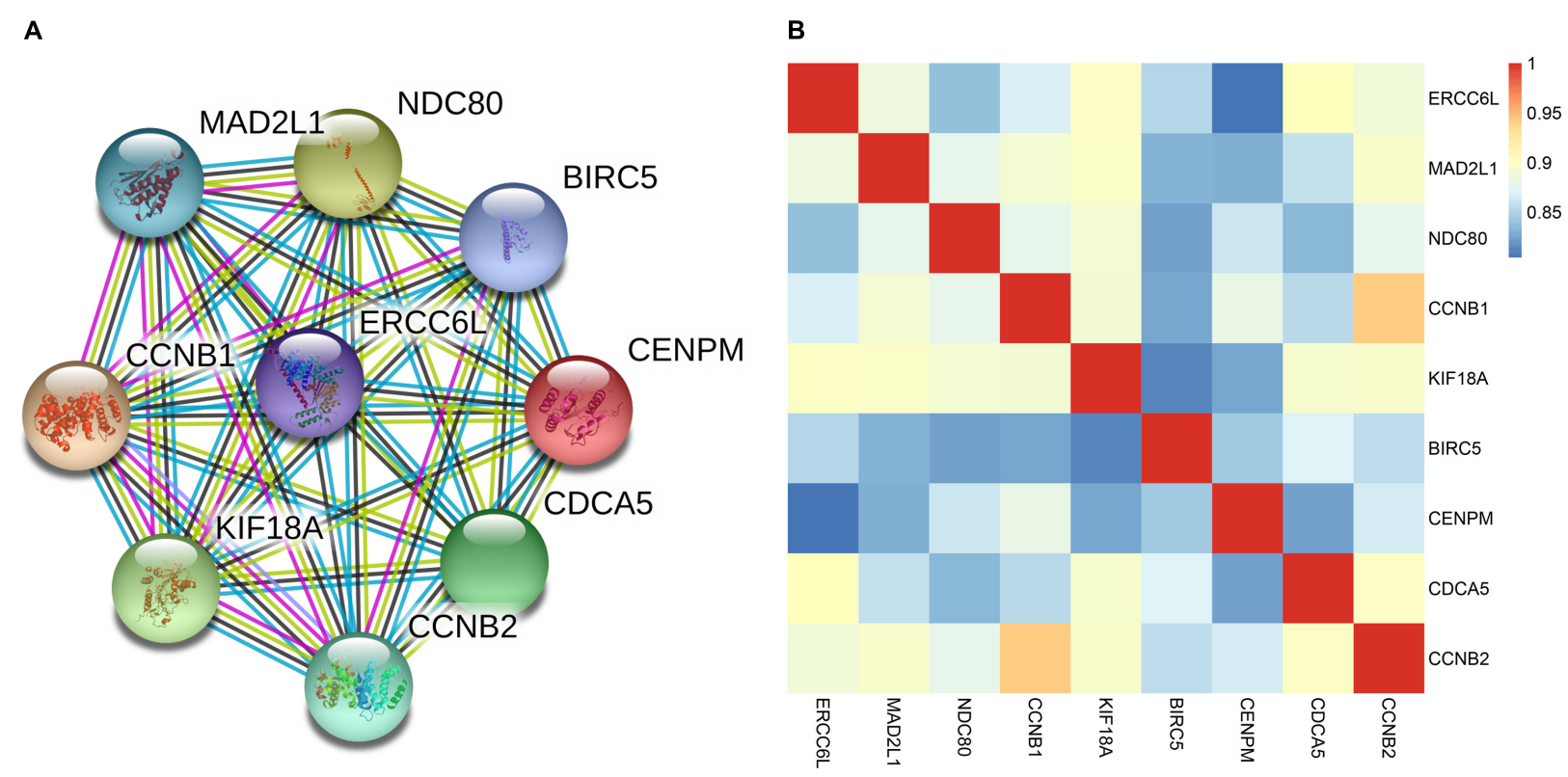

FIGURE 5 | Correlation of ERCC6L with other key genes in NB. (A) The protein-protein interaction network of ERCC6L and other key genes; (B) Pearson correlation coefficient of 9 genes in NB. 
A

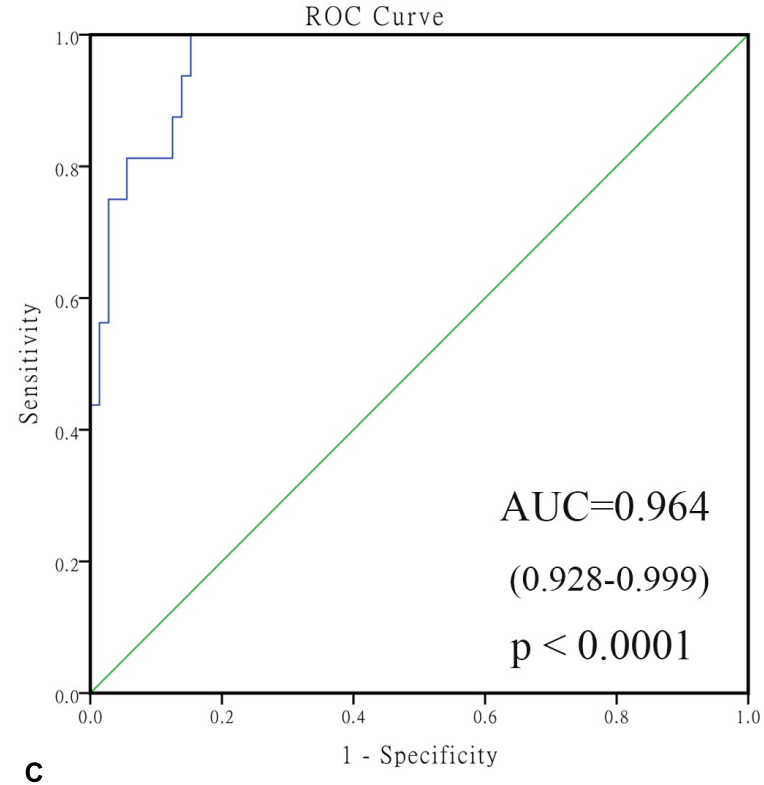

C

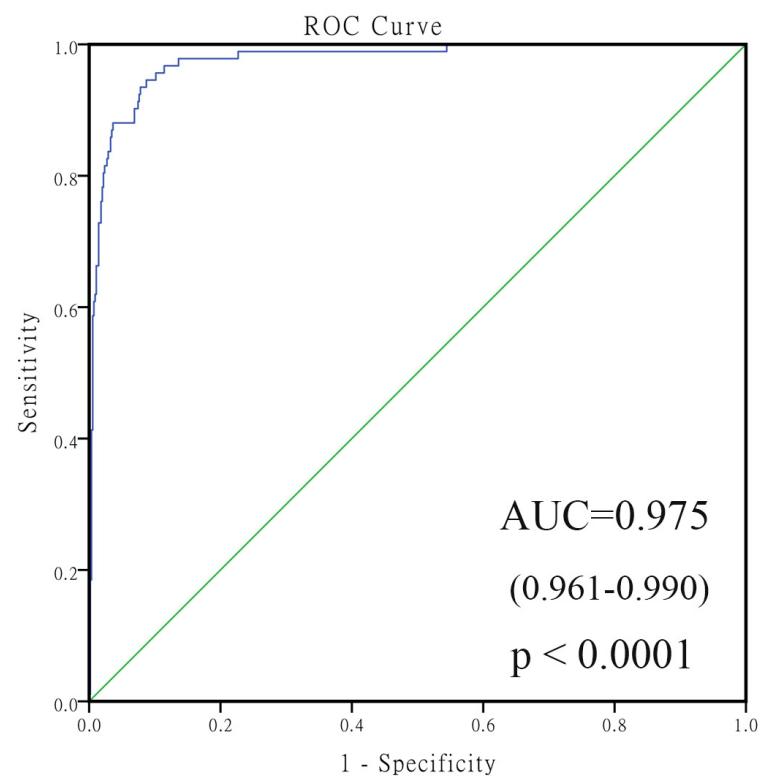

B
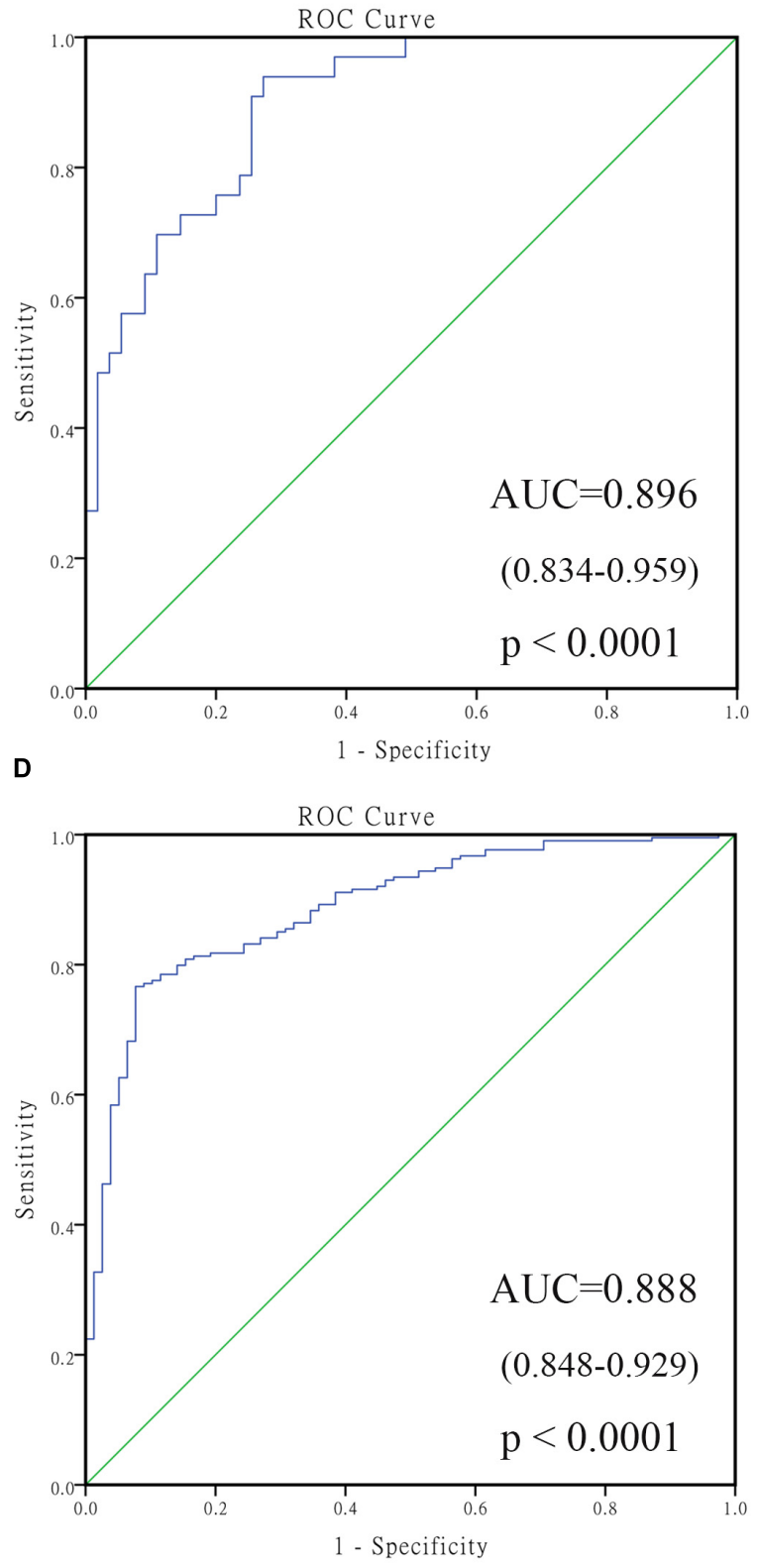

FIGURE 6 | The receiving operating characteristic curve (ROC) of validated datasets. (A) ROC of MYCN status in GSE16476; (B) ROC of vital status in GSE16476; (C) ROC of MYCN status in GSE45480; (D) ROC of stage 4 vs. stage 4s in GSE45480.

outcomes. However, it is difficult to develop a satisfactory prognostic model, which can be ascribed to extreme cancer complexity. MYCN amplification, ALK mutation (Barone et al., 2013; Lambertz et al., 2015), or other molecular biomarkers (Egler et al., 2008; Pugh et al., 2013; Zage et al., 2013) have provided researchers with some hints, yet more studies are still needed. In particular, current researches mainly focused on how to reduce the treatment-associated side effect in patients with good prognosis, and how to provide more effective treatment for patients with poor prognosis patients (Whittle et al., 2017). This requires researchers to find more markers that distinguish patients with good prognosis and poor prognosis. Our study identified a four-gene signature in predicting prognosis and discriminating other groups, which had attained satisfactory performance.

Multivariate Cox regression analysis showed that the four-gene signature and INSS stage are all independent prognostic indicators. The two features are highly correlated and have a prognostic significance, this indicated that there is a part of coincident information between them, but the signature also has its own independent prognostic information, and it contributes more to the prognosis analysis of NB patients 
according to the $p$-value. In addition, in order to support our results, we removed the INSS stage and applied other features to analyze again. The results showed that the signature was still an independent prognostic indicator, further validating the effect of our signature. At the same time, survival analysis of the four-gene signature in stage 4 and MYCN non-amplification patients, and age of $<18$ months was also performed, and the results indicated that the high risk score group had significantly inferior outcomes. In conclusion, our four-gene signature might help clinicians to choose the treatment regimens and aid them to predict patient prognosis in a similar situation. We got similar results in the independent dataset GSE16476, which can support the robustness of our signature in predicting the prognosis in NB patients.

Of the four genes, AHCY has been reported to be a direct target of MYCN, which predicts poor prognosis in NB (Chayka et al., 2015). NCAN has been confirmed to promote the malignant phenotypes in NB cells (Su et al., 2017). ERCC Excision Repair 6 Like (ERCC6L, Spindle Assembly Checkpoint Helicase) is a protein-coding gene, also known as $\mathrm{PICH}$, its related pathways include cell cycle and mitosis. $\mathrm{Pu}$ et al. had found that higher ERCC6L expression was notably associated with inferior outcomes in breast and kidney cancers, and silencing of ERCC6L would inhibit the growth of breast and kidney cancer cells. Besides, their further studies considered that ERCC6L might affect the cell cycle via RAB31-MAPF-CDK, so as to promote cancer cell proliferation ( $\mathrm{Pu}$ et al., 2017). Albers et al. showed that loss of PICH results in DNA damage, P53 activation, and embryonic development impaired (Albers et al., 2018), which indicated that PICH may play an important role in embryonic lethality and tumorigenesis. In our study, univariate and multivariate Cox regression analyses revealed that ERCC6L could be an independent prognostic factor of overall survival and event-free survival $(p<0.0001)$. Typically, the AUC of ERCC6L alone in predicting different groups was higher than $0.75(p$ $<0.0001)$.

In order to find the potential function of ERCC6L, we have constructed a PPI network and calculated the PCC of ERCC6L with other key genes which may interact with ERCC6L, eight

\section{REFERENCES}

Albers, E., Sbroggio, M., Pladevall-Morera, D., Bizard, A. H., Avram, A., Gonzalez, P., et al. (2018). Loss of PICH results in chromosomal instability, p53 activation, and embryonic lethality. Cell Rep. 24, 3274-3284. doi: 10.1016/j. celrep.2018.08.071

Attiyeh, E. F., and Maris, J. M. (2015). Identifying rare events in rare diseases. Clin. Cancer Res. 21, 1782-1785.

Bader, G. D., and Hogue, C. (2003). An automated method for finding molecular complexes in large protein interaction networks. BMC Bioinformatics 4:2.

Barone, G., Anderson, J., Pearson, A. D. J., Petrie, K., and Chesler, L. (2013). New strategies in neuroblastoma: therapeutic targeting of MYCN and ALK. Clin. Cancer Res. 19, 5814-5821. doi: 10.1158/1078-0432.CCR-13-0680

Beckers, A., Van Peer, G., Carter, D. R., Gartlgruber, M., Herrmann, C., Agarwal, S., et al. (2015). MYCN-driven regulatory mechanisms controlling LIN28B in neuroblastoma. Cancer Lett. 366, 123-132. doi: 10.1016/j.canlet.2015.06.015

Bénard, J., Raguénez, G., Kauffmann, A., Valent, A., Ripoche, H., Joulin, V., et al. (2008). MYCN-non-amplified metastatic neuroblastoma with good prognosis genes had been identified that had a close relationship with it. Most of them were involved in the cell cycle pathway. Importantly, of the eight genes, MAD2L (Gogolin et al., 2013), CCNB1 (Liu et al., 2013; Schwermer et al., 2015), and BIRC5 (Lamers et al., 2011; Hagenbuchner et al., 2016) have been proven play important roles in NB development and metastasis. Taken together, we considered ERCC6L may play an important role in $\mathrm{NB}$, biological experiments are needed for further study of its mechanism.

\section{AUTHOR CONTRIBUTIONS}

YL conceived of and directed the project. XZ designed the study, analyzed the data, wrote the manuscript. HL revised the manuscript critically for important intellectual content. YZ collected data and samples. LW reviewed the data. All authors have read and approved the final manuscript for publication.

\section{FUNDING}

This research was supported by the National Natural Science Foundation of China (Grant Nos. 61471181 and 81702966) and the Natural Science Foundation of Jilin Province (Grant Nos. 20140101194JC and 20150101056JC).

\section{ACKNOWLEDGMENTS}

We wish to acknowledge Chao Lu for his help in processing the data with the random forest algorithm.

\section{SUPPLEMENTARY MATERIAL}

The Supplementary Material for this article can be found online at: https://www.frontiersin.org/articles/10.3389/fgene. 2018.00589/full\#supplementary-material

and spontaneous regression: a molecular portrait of stage 4S. Mol. Oncol. 2, 261-271. doi: 10.1016/j.molonc.2008.07.002

Bosse, K. R., and Maris, J. M. (2016). Advances in the translational genomics of neuroblastoma: from improving risk stratification and revealing novel biology to identifying actionable genomic alterations. Cancer 122, 20-33. doi: 10.1002/ cncr.29706

Brodeur, G. M., and Bagatell, R. (2014). Mechanisms of neuroblastoma regression. Nat. Rev. Clin. Oncol. 11, 704-713. doi: 10.1038/nrclinonc.2014.168

Ceccaldi, R., Sarangi, P., and D'Andrea, A. D. (2016). The fanconi anaemia pathway: new players and new functions. Nat. Rev. Mol. Cell Biol. 17:337. doi: $10.1038 / \mathrm{nrm} .2016 .48$

Chayka, O., D’Acunto, C. W., Middleton, O., Arab, M., and Sala, A. (2015). Identification and pharmacological inactivation of the MYCN gene network as a therapeutic strategy for neuroblastic tumor cells. J. Biol. Chem. 290, 2198-2212. doi: $10.1074 /$ jbc.M114.624056

Chin, C. H., Chen, S. H., Wu, H. H., Ho, C. W., Ko, M. T., and Lin, C. Y. (2014). cytoHubba: identifying hub objects and sub-networks from complex interactome. BMC Syst. Biol. 8(Suppl. 4):S11. doi: 10.1186/1752-0509-8-S4-S11 
Diede, S. J. (2014). Spontaneous regression of metastatic cancer: learning from neuroblastoma. Nat. Rev. Cancer 14, 71-72. doi: 10.1038/nrc3656

Domingo-Fernandez, R., Watters, K., Piskareva, O., Stallings, R. L., and Bray, I. (2013). The role of genetic and epigenetic alterations in neuroblastoma disease pathogenesis. Pediatr. Surg. Int. 29, 101-119. doi: 10.1007/s00383-012-3239-7

Egler, R. A., Burlingame, S. M., Nuchtern, J. G., and Russell, H. V. (2008). Interleukin-6 and soluble interleukin-6 receptor levels as markers of disease extent and prognosis in neuroblastoma. Clin. Cancer Res. 14, 7028-7034. doi: 10.1158/1078-0432.CCR-07-5017

Fischer, M., Oberthuer, A., Brors, B., Kahlert, Y., Skowron, M., Voth, H., et al. (2006). Differential expression of neuronal genes defines subtypes of disseminated neuroblastoma with favorable and unfavorable outcome. Clin. Cancer Res. 12, 5118-5128.

Gogolin, S., Batra, R., Harder, N., Ehemann, V., Paffhausen, T., Diessl, N., et al. (2013). MYCN-mediated overexpression of mitotic spindle regulatory genes and loss of p53-p21 function jointly support the survival of tetraploid neuroblastoma cells. Cancer Lett. 331, 35-45. doi: 10.1016/j.canlet.2012. 11.028

Hagenbuchner, J., Kiechl-Kohlendorfer, U., Obexer, P., and Ausserlechner, M. J. (2016). BIRC5/Survivin as a target for glycolysis inhibition in high-stage neuroblastoma. Oncogene 35, 2052-2061. doi: 10.1038/onc. 2015.264

Huang, M., and Weiss, W. A. (2013). Neuroblastoma and MYCN. Cold Spring Harbor Perspect. Med. 3:a014415. doi: 10.1101/cshperspect.a014415

Kanehisa, M., Furumichi, M., Mao, T., Sato, Y., and Morishima, K. (2017). KEGG: new perspectives on genomes, pathways, diseases and drugs. Nucleic Acids Res. 45, D353-D361. doi: 10.1093/nar/gkw1092

Kelemen, L. E., Terry, K. L., Goodman, M. T., Webb, P. M., Bandera, E. V., Mcguire, V., et al. (2014). Consortium analysis of gene and gene-folate interactions in purine and pyrimidine metabolism pathways with ovarian carcinoma risk. Mol. Nutr. Food Res. 58, 2023-2035. doi: 10.1002/mnfr. 201400068

Lambertz, I., Kumps, C., Claeys, S., Lindner, S., Beckers, A., Janssens, E., et al. (2015). Upregulation of MAPK negative feedback regulators and RET in Mutant ALK neuroblastoma: implications for targeted treatment. Clin. Cancer Res. 21, 3327-3339. doi: 10.1158/1078-0432.CCR-14-2024

Lamers, F., van der Ploeg, I., Schild, L., Ebus, M. E., Koster, J., Hansen, B. R., et al. (2011). Knockdown of survivin (BIRC5) causes apoptosis in neuroblastoma via mitotic catastrophe. Endocr. Relat. Cancer 18, 657-668. doi: 10.1530/ERC-110207

Liao, X., Zhu, G., Huang, R., Yang, C., Wang, X., Huang, K., et al. (2018). Identification of potential prognostic microRNA biomarkers for predicting survival in patients with hepatocellular carcinoma. Cancer Manag. Res. 10, 787-803. doi: 10.2147/CMAR.S161334

Liu, Z., Rader, J., He, S., Phung, T., and Thiele, C. J. (2013). CASZ1 inhibits cell cycle progression in neuroblastoma by restoring $\mathrm{pRb}$ activity. Cell Cycle 12, 2210-2218. doi: $10.4161 / \mathrm{cc} .25265$

Liu, Z., and Thiele, C. J. (2017). When LMO1 Meets MYCN, Neuroblastoma Is Metastatic. Cancer Cell 32, 273-275. doi: 10.1016/j.ccell.2017.08.014

Maris, J. M., Hogarty, M. D., Bagatell, R., and Cohn, S. L. (2007). Neuroblastoma. Lancet 369, 2106-2120.

Otto, T., and Sicinski, P. (2017). Cell cycle proteins as promising targets in cancer therapy. Nat. Rev. Cancer 17, 93-115. doi: 10.1038/nrc.2016.138

Pinto, N. R., Applebaum, M. A., Volchenboum, S. L., Matthay, K. K., London, W. B., Ambros, P. F., et al. (2015). Advances in risk classification and treatment strategies for neuroblastoma. J. Clin. Oncol. 33, 3008-3017.

Prentice, R. L. (1992). "Introduction to Cox (1972) regression models and lifetables," in Breakthroughs in Statistics. Springer Series in Statistics (Perspectives in Statistics), eds S. Kotz and N. L. Johnson (New York, NY: Springer).

$\mathrm{Pu}, \mathrm{S}$. Y., Yu, Q., Wu, H., Jiang, J. J., Chen, X. Q., He, Y. H., et al. (2017). ERCC6L, a DNA helicase, is involved in cell proliferation and associated with survival and progress in breast and kidney cancers. Oncotarget 8, 42116-42124. doi: 10.18632/oncotarget.14998

Pugh, T. J., Morozova, O., Attiyeh, E. F., Asgharzadeh, S., Wei, J. S., Auclair, D., et al. (2013). The genetic landscape of high-risk neuroblastoma. Nat. Genet. 45, 279-284. doi: $10.1038 / \mathrm{ng} .2529$
Ritchie, M. E., Phipson, B., Wu, D., Hu, Y., Law, C. W., Shi, W., et al. (2015). limma powers differential expression analyses for RNA-sequencing and microarray studies. Nucleic Acids Res. 43:e47. doi: 10.1093/nar/gkv007

Rubie, H., De Bernardi, B., Gerrard, M., Canete, A., Ladenstein, R., Couturier, J., et al. (2011). Excellent outcome with reduced treatment in infants with nonmetastatic and unresectable neuroblastoma without MYCN amplification: results of the prospective INES 99.1. J. Clin. Oncol. 29, 449-455. doi: 10.1200/ JCO.2010.29.5196

Salazar, B. M., Balczewski, E. A., Ung, C. Y., and Zhu, S. (2016). Neuroblastoma, a paradigm for big data science in pediatric oncology. Int. J. Mol. Sci. 18:E37. doi: 10.3390/ijms18010037

Sausen, M., Leary, R. J., Jones, S., Wu, J., Reynolds, C. P., Liu, X., et al. (2013). Integrated genomic analyses identify ARID1A and ARID1B alterations in the childhood cancer neuroblastoma. Nat. Genet. 45, 12-17. doi: 10.1038/ng.2493

Schwermer, M., Lee, S., Köster, J., van Maerken, T., Stephan, H., and Eggert, A. (2015). Sensitivity to cdk1-inhibition is modulated by p 53 status in preclinical models of embryonal tumors.pdf. Oncotarget 6, 15425-15435.

Shannon, P., Markiel, A., Ozier, O., Baliga, N. S., Wang, J. T., Ramage, D. et al. (2003). Cytoscape: a software environment for integrated models of biomolecular interaction networks. Genome Res. 13:2498.

Stafman, L. L., and Beierle, E. A. (2016). Cell proliferation in neuroblastoma. Cancers 8:E13. doi: 10.3390/cancers8010013

Strobl, C., Boulesteix, A. L., Zeileis, A., and Hothorn, T. (2007). Bias in random forest variable importance measures: illustrations, sources and a solution. $B M C$ Bioinformatics 8:25.

Su, Z. D., Kishida, S., Tsubota, S., Sakamoto, K., Cao, D. L., Kiyonari, S., et al. (2017). Neurocan, an extracellular chondroitin sulfate proteoglycan, stimulates neuroblastoma cells to promote malignant phenotypes. Oncotarget 8 , 106296-106310. doi: 10.18632/oncotarget.22435

Taggart, D. R., London, W. B., Schmidt, M. L., DuBois, S. G., Monclair, T. F., Nakagawara, A., et al. (2011). Prognostic value of the stage 4S metastatic pattern and tumor biology in patients with metastatic neuroblastoma diagnosed between birth and 18 months of age. Journal Of Clinical Oncology 29, 4358-4364. doi: 10.1200/JCO.2011.35.9570

The Gene Ontology Consortium. (2017). Expansion of the gene ontology knowledgebase and resources. Nucleic Acids Res. 45, D331-D338. doi: 10.1093/ nar/gkw1108

Ward, E., DeSantis, C., Robbins, A., Kohler, B., and Jemal, A. (2014). Childhood and adolescent cancer statistics, 2014. CA Cancer J. Clin. 64, 83-103. doi: $10.3322 /$ caac. 21219

Whittle, S. B., Smith, V., Doherty, E., Zhao, S., McCarty, S., and Zage, P. E. (2017). Overview and recent advances in the treatment of neuroblastoma. Expert Rev. Anticancer Ther. 17, 369-386. doi: 10.1080/14737140.2017.1285230

Williams, G. H., and Stoeber, K. (2012). The cell cycle and cancer. J. Pathol. 226, 352-364.

Zage, P. E., Sirisaengtaksin, N., Liu, Y., Gireud, M., Brown, B. S., Palla, S., et al. (2013). UBE4B levels are correlated with clinical outcomes in neuroblastoma patients and with altered neuroblastoma cell proliferation and sensitivity to epidermal growth factor receptor inhibitors. Cancer 119, 915-923. doi: 10.1002/ cncr. 27785

Zhou, M., Zhao, H., Wang, Z., Cheng, L., Yang, L., Shi, H., et al. (2015). Identification and validation of potential prognostic lncRNA biomarkers for predicting survival in patients with multiple myeloma. J. Exp. Clin. Cancer Res. $34: 102$

Conflict of Interest Statement: The authors declare that the research was conducted in the absence of any commercial or financial relationships that could be construed as a potential conflict of interest.

Copyright () 2018 Zhong, Liu, Liu, Zhang, Wang and Zhang. This is an open-access article distributed under the terms of the Creative Commons Attribution License (CC BY). The use, distribution or reproduction in other forums is permitted, provided the original author(s) and the copyright owner(s) are credited and that the original publication in this journal is cited, in accordance with accepted academic practice. No use, distribution or reproduction is permitted which does not comply with these terms. 